\title{
INSTABILITY OF THE EXISTENCE OF NON-ABELIAN VECTOR POTENTIALS
}

\author{
David N. WILLIAMS \\ Randall Laboratory of Physics, The University of Michigan, Ann Arbor, MI 48109, USA
}

Received 13 March 1978

\begin{abstract}
We show the instability of the existence of vector potentials against variations of the field tensor in regions where it is "strongly non-abelian".
\end{abstract}

When does a classical, non-abelian Yang-Mills field tensor $F_{\mu \nu}$ have a vector potential $A$ ? The answer to this question turns out to be tied up with the nonuniqueness theorem for vector potentials. The nonuniqueness theorem began with a counterexample of Wu and Yang [1], which showed there are non-gaugeequivalent $A$ 's that give the same non-abelian $F$, and some work of Deser and Teitelboim [2], and culminated in the works of Deser and Wilczek [3], Calvo [4], Roskies [5], Halperm [6], and Hsia, referred to in ref. [5], which showed that a necessary condition for uniqueness was the nonvanishing of the determinant of the matrix $M$ that we now define.

For each $x \in \mathrm{R}^{4}$, let $F_{\mu \nu}(x)=-F_{\nu \mu}(x)$ be a hermitean, finite-dimensional matrix, belonging to a representation of the Lie algebra of a compact, semisimple Lie group. The tensor indices are four-vector indices. Let $F^{\mathrm{D}}$ bc the usual, second-rank tensor dual to $F$. Let $n$ be the dimension of the Lie algebra. Then $M$ is the $4 n \times 4 n$ matrix

$M_{(\mu a)(\nu b)}=f_{a c b}\left(F_{\mu \nu}^{\mathrm{D}}\right)_{c}$,

where $f_{a c b}$ are the Lie algebra structure constants, and $\left(F^{\mathrm{D}}\right)_{c}$ an appropriate Lie algebra component of $F^{\mathrm{D}}$.

It is still unknown how to classify the relations between $F$ and its vector potential $A$, where

$F_{\mu \nu}=\partial_{\mu} A_{\nu}-\partial_{\nu} A_{\mu}-\mathrm{i}\left[A_{\mu}, A_{\nu}\right]$,

at points where $\operatorname{det} M=0$. An interesting recent paper by Kazama and Lee [7] studies the determinant for instantons, and shows that the zeroes of $\operatorname{det} M$ have topological significance.

The uniqueness theorem states that, at points where the determinant is nonzero (we call these strongly non-abelian points), the Bianchi identity may be solved uniquely for $A=A\left(F^{\mathrm{D}}\right)$, simply by inverting the matrix $M$ against $\partial_{\mu} F_{\mu \nu}^{\mathrm{D}}$. Deser and Wilczek [3] give some remarks on the different role played by the Bianchi identity for abelian versus non-abelian $F$ 's. We want to mention the basic point that in the abelian theory, the Bianchi identity for $F$ guarantees the existence of an $A$, while in the non-abelian theory, the Bianchi identity cannot even be stated in terms of $F$ alone.

In this note, we state a theorem which shows that the existence of a vector potential at strongly nonabelian points is special. Of course the existence of a vector potential is also special in the abelian theory; the Bianchi identity must be satisfied; but here we need different tools to reach that conclusion.

The following theorem is stated for euclidean fields with finite action. As we discuss later, finite action is not essential; the result is basically local, and holds locally for Minkowski fields as well. The theorem states that, for regular $F$ 's, the property of having an $A$ is destroyed by regular variations of $F$ in regions where it is strongly non-abelian.

Theorem. Let $F$ have finite action and be derivable from a sufficiently regular vector potential. Let $\operatorname{det} M$, defined by eq. (1), be nonvanishing on an open set in $\mathbf{R}^{4}$. Let $F^{\prime}=F+\delta F$, where the variation has the following properties: 
(i) $\delta F$ is sufficiently regular;

(ii) $\delta F=0$ outside a bounded region and on a closed neighborhood of the zeroes of det $M$;

(iii) $\operatorname{det}(M+\delta M) \neq 0$, except where $\operatorname{det} M=0$;

(iv) $Q(F) \equiv \frac{1}{2} \int \mathrm{d} x \operatorname{Tr} F_{\mu \nu} F_{\mu \nu}^{\mathrm{D}} \neq Q(F+\delta F)$.

Then there is a set of non-zero Lebesgue measure on which $F^{\prime}$ has no vector potential.

Proof. Suppose $F^{\prime}$ is derivable from a vector potential $B$. Let $A$ be the regular vector potential that gives $F$.

(i) The first step is to show that $B$ may be chosen to be regular, and to coindice with $A$ outside a bounded region in $\mathbf{R}^{4}$. By hypothesis, $F^{\prime}=F$ outside a bounded region $U$ in the interior of the set where $\operatorname{det} M \neq 0$. Outside $U$, we choose $B=A$. Inside $U$, $\operatorname{det} M^{\prime}$ is nonvanishing and regular. From the uniqueness theorem, the Bianchi identity may be solved there uniquely for $B$ in terms of $F^{\prime}$; and the solution will be regular (one simply has to invert the matrix $M^{\prime}$ against the ordinary divergence of $F^{\prime D}$ ). Moreover, $B$ patches smoothly onto $A$ in the region where $F^{\prime}=F$, because that region extends into the interior of the set where $\operatorname{det} M \neq 0$, where the solution for the vector potential is unique, and where everything is regular.

(ii) The next step is to compute

$Q\left(F^{\prime}\right)=\int \mathrm{d} x \partial \cdot S$,

where we use the familiar identity

$S=\frac{1}{2} \epsilon_{\mu \nu \lambda \rho} \operatorname{Tr}\left(B_{\nu} \partial_{\lambda} B_{\rho}-\frac{2}{3}\right.$ i $\left.B_{\nu} B_{\lambda} B_{\rho}\right)$.

Since the action is finite for $F$, it is also finite for $F^{\prime}$; and the four-dimensional integral $Q\left(F^{\prime}\right)$ converges absolutely, by the Schwartz inequality, as usual. Moreover, $S(B)$ and $\partial \cdot S(B)$ are sufficiently regular, because $B$ is, so that the divergence theorem gives $Q\left(F^{\prime}\right)$ as an integral over the surface at infinity, with no contributions from finite points. But on such a large sphere, $B=A$; and so $Q\left(F^{\prime}\right)=Q(F)$, contrary to the hypothesis. It is therefore impossible that $F^{\prime}=F(B)$ everywhere.

(iii) Finally, we show that the set where $F^{\prime}$ has no vector potential is nontrivial. The only points where $F^{\prime}$ has no vector potential are points where $\delta F \neq 0$, where the only possible vector potential is $B$. Let $V \subset U$ be the set where $F^{\prime} \neq F(B)$. If this set has zero Lebesgue measure, then so does its closure $\bar{V}$. Therefore $\bar{V}$ has an empty interior. At points outside $\bar{V}$, $F^{\prime}=F(B)$. Since $F^{\prime}$ and $B$ are both sufficiently regular everywhere, including $\bar{V}$, and since $\bar{V}$ has no interior, we conclude by continuity that $F^{\prime}=F(B)$ also on $\bar{V}$, a contradiction. Therefore $V$ has nonzero Lebesgue measure. That completes the proof.

To summarize the essential idea, the existence of the vector potential for regular potentials is unstable against regular variations of $F$ at strongly non-abelian points, because any vector potential is unique there, and because the topological charge is stable against regular, local variations of $A$, but unstable against local variations of $F$.

A situation in the abelian theory analogous to the nonzero measure property of the region where the vector potential fails to exist would be a magnetic field with a magnetic charge source extended over a finite region.

Remark 1 . From the proof, "sufficiently regular" means regular enough to get no contributions from finite points in the divergence theorem, and to admit the continuity argument in step (iii) of the proof. The latter condition is that $B$ and $\partial B$ be continuous. If $\partial \partial B$ also exists almost everywhere, the divergence argument works, because then $S$ and $\partial \cdot S$ are continuoy

Remark 2. Finite action is not essential, because if $F$ comes from a regular $A$, we could apply the theorem for finite action to $F(\eta A)$, where $\eta$ is a smooth function equal to one in a bounded region of interest, and zero outside a larger, bounded region. Or, we could repeat the argument for $F$ and $A$ defined only on a bounded region with a good surface, with variations $\delta F=0$ on the surface. In either form, the theorem can be proved locally also for Minkowski fields.

Remark 3. The theorem certainly applies to the one-instanton, selfdual solution of the Yang-Mills field equation in the B.P.S.T. form [8], which has a $\mathrm{C}^{\infty}$ vector potential, and for which $\operatorname{det} M$ is everywhere nonvanishing [3]. Then it is sufficient to choose $F^{\prime}$ $=(1+\alpha) F$, where $\alpha$ is non-negative, smooth, nonvanishing in some open set, and of bounded support, to get (selfdual) field tensors having no vector potential in nontrivial regions. Indeed, Kazama and Lee [7] have shown that there are gauges in which all of the 't Hooft instantons [9] have regular vector potentials; and although the regions of nonvanishing determinant have not been completely mapped out in general, a similar discussion applies to variations of their field tensors.

Remark 4. There remains the question of how to 
characterize the $F$ 's that have $A$ 's. We wonder whether the following condition might not be necessary and sufficient:

$\left[\operatorname{det} M\left(F^{\mathrm{D}}\right)\right]^{2}\left\{F-F\left[A\left(F^{\mathrm{D}}\right)\right]\right\}=0$.

This is to be regarded as a non-linear equation for $F$. It is a necessary and sufficient equation for $F$ to have an $A$ at strongly non-abelian points. When $\operatorname{det} M=0$, the condition becomes

$$
\left[\operatorname{det} M\left(F^{\mathrm{D}}\right)\right]^{2} F\left[A\left(F^{\mathrm{D}}\right)\right]=0 .
$$

This equation is well defined, because the adjoint matrix $M^{-1} \operatorname{det} M$ is well defined, and may be used to define $A\left(F^{\mathrm{D}}\right)$ det $M\left(F^{\mathrm{D}}\right)$. A plausibility argument for the necessity of this condition is that $A$ 's giving zero determinants might be approximable by $A$ 's with nonzero determinants, whereupon necessity follows by continuity. One ought to verify necessity by ordinary algebra. A possible difficulty with the sufficiency of eq. (6) is that there might be situations where it becomes overconstraining. For example, the null space of the adjoint matrix $M^{-1} \operatorname{det} M$ might combine with the vanishing of the component of $\partial \cdot F^{\mathrm{D}}$ on the orthogonal complement of that null space to make $M^{-1} \operatorname{det} M \partial \cdot F^{\mathrm{D}}=0$ on an open set for some class of $F$ 's, whereupon a short calculation shows that eq. (6) is automatically satisfied. As a simple case, all $F$ 's such that $\partial \cdot F^{\mathrm{D}}=0$ and $\operatorname{det} M=0$ on an open set would have to have a vector potential for eq. (6) to be a sufficient condition.

\section{References}

[1] T.T. Wu and C.N. Yang, Phys. Rev. D12 (1975) 3843.

[2] S. Deser and C. Teitelboim, Phys. Rev. D13 (1976) 1592.

[3] S. Deser and F. Wilczek, Phys. Lett. 65B (1976) 391.

[4] M. Calvo, Phys. Rev. D15 (1977) 1733.

[5] R. Roskies, Phys. Rev. D15 (1977) 1731.

[6] M.B. Halpern, Phys. Rev. D16 (1977) 1798.

[7] Y. Kazama and C. Lee, Topological properties of singular pseudoparticle solutions, Univ. of Michigan, Randall Lab. of Physics preprint (1977).

[8] A. Belavin, A. Polyakov, A. Schwartz and Y. Tyupkin, Phys. Lett. 59B (1975) 85.

[9] G. 't Hooft, Coral Gables Conf. (1977). 